Check for updates

Cite this: RSC Adv., 2017, 7, 37310

\title{
Selective and confined growth of transition metal dichalcogenides on transferred graphene $\uparrow$
}

\author{
Fei Lu, (D) a Arka Karmakar, ${ }^{a}$ Simran Shahi ${ }^{a}$ and Erik Einarsson (iD *ab
}

We demonstrate confinement of CVD grown $\mathrm{MoS}_{2}$ to a patterned graphene area, forming a vertically stacked 2D heterostructure. The CVD-grown graphene had been transferred onto a Si wafer and patterned using photolithography. Raman mapping and spectral analysis reveal few-layer $\mathrm{MoS}_{2}$ grew selectively on graphene regions, and not on the surrounding $\mathrm{SiO}_{2}$ substrate surface. We also report CVD growth of $\mathrm{WS}_{2}$ directly on transferred graphene. Unlike $\mathrm{MoS}_{2}$, no few-layer regions were found; the $\mathrm{WS}_{2}$ was found to be either monolayer or at least five layers (bulk). The $W_{2}$ coverage was only partial, but selectivity to graphene is apparent. These findings have the potential to significantly advance fabrication of vertical 2D heterostructures and related devices, and suggest the selective growth on graphene may be applicable to TMDCs in general.

Received 14th July 2017

Accepted 19th July 2017

DOI: 10.1039/c7ra07772f

rsc.li/rsc-advances
Here we report CVD growth of molybdenum disulfide $\left(\mathrm{MoS}_{2}\right)$ directly atop, and laterally confined by, an underlying graphene pattern. We also report CVD synthesis of tungsten disulfide $\left(\mathrm{WS}_{2}\right)$ directly on graphene. While the $\mathrm{WS}_{2}$ coverage was not complete, growth was selective to graphene and did not occur on the $\mathrm{SiO}_{2}$ substrate surface. These results are an important step toward the ability to pre-define the growth location of $2 \mathrm{D}$ heterostructures by patterned graphene templates.

\section{Experimental}

To produce vertically stacked TMDC-graphene heterostructures, we first synthesized graphene at $1000{ }^{\circ} \mathrm{C}$ on copper foil using low-pressure chemical vapor deposition (CVD). We then transferred the graphene onto a silicon substrate with a $285 \mathrm{~nm}$ oxide layer $\left(\mathrm{SiO}_{2} / \mathrm{Si}\right)$. This was done using an unpublished variation on a widely used wet process, ${ }^{\mathbf{1 4}, 15}$ in which we use a copolymer layer in addition to a layer of poly(methyl methacrylate) (PMMA). We then used the graphene-on- $\mathrm{SiO}_{2} / \mathrm{Si}$ as a substrate for TMDC growth $\left(\mathrm{MoS}_{2}\right.$ and $\left.\mathrm{WS}_{2}\right)$. In the case of $\mathrm{MoS}_{2}$ growth, we patterned the transferred graphene prior to CVD using standard photolithography techniques.

We grew $\mathrm{MoS}_{2}$ by CVD in a tube furnace at $700{ }^{\circ} \mathrm{C}$ and atmospheric pressure. We prepared the molybdenum source by dispersing $\mathrm{MoO}_{3}$ powder in ethanol, and then dropping the dispersion onto a $5 \times 5 \mathrm{~mm}$ piece of silicon wafer. ${ }^{16}$ After the ethanol evaporated, approximately $10 \mathrm{mg}$ of $\mathrm{MoO}_{3}$ remained. We then placed the patterned graphene-on- $\mathrm{SiO}_{2} / \mathrm{Si}$ centered directly above the $\mathrm{MoO}_{3}$ source at a distance of approximately 7 $\mathrm{mm}$, with the patterned graphene side facing toward the $\mathrm{MoO}_{3}$. The sulfur source was placed $30 \mathrm{~cm}$ upstream from the $\mathrm{MoO}_{3}$ source (10 cm outside the furnace), and heated separately using a heating belt. Prior to CVD, we purged air from the system by 


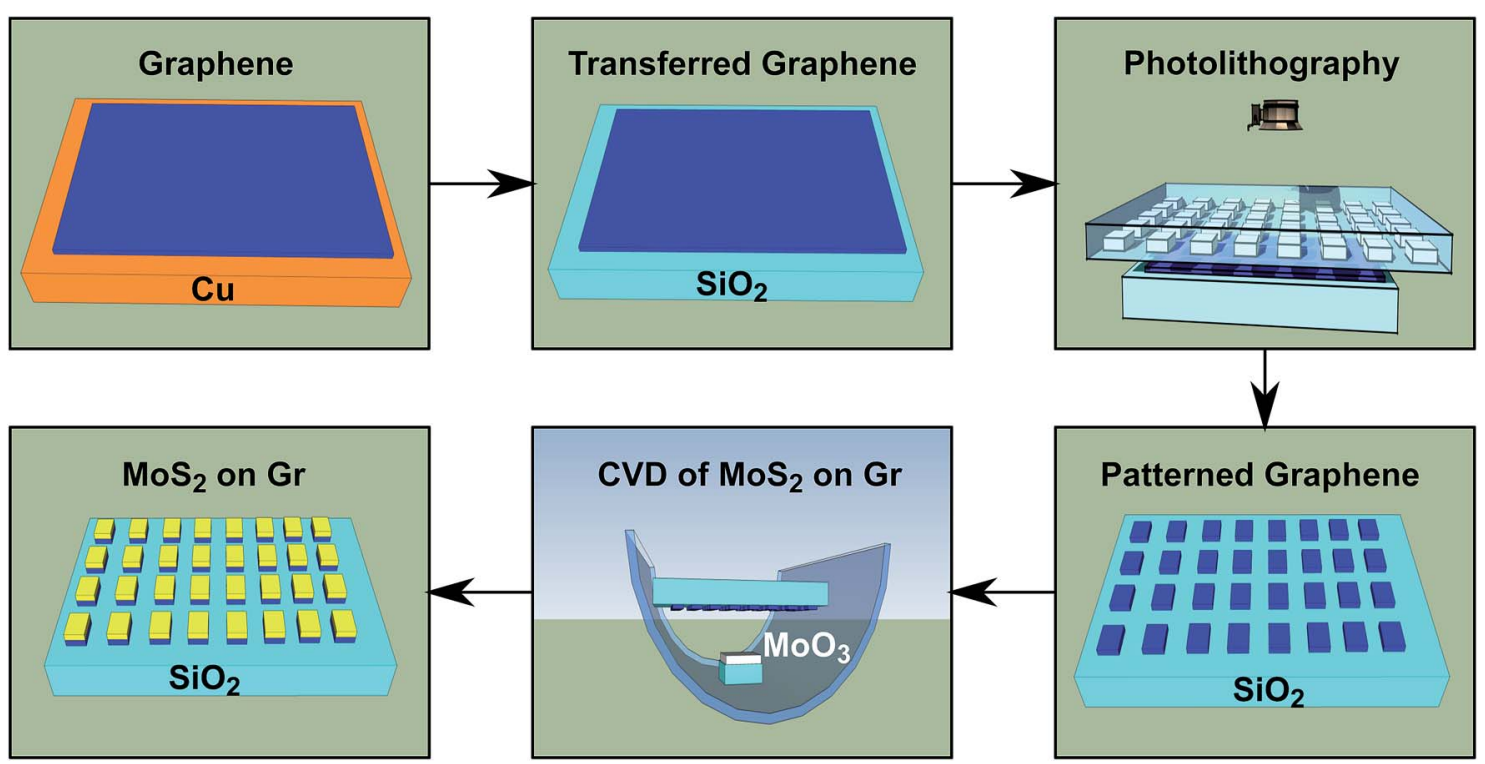

Fig. 1 Illustration of processing steps (clockwise from top left) resulting in localized CVD growth of $M_{0} S_{2}$ on patterned graphene.

evacuating the quartz tube (26 $\mathrm{mm}$ ID) and refilling to atmospheric pressure using pure Ar gas. We continued to supply Ar at a flow rate of $150 \mathrm{sccm}$ throughout the entire CVD process. We increased the furnace temperature by $20^{\circ} \mathrm{C}$ per minute until reaching $700{ }^{\circ} \mathrm{C}$, at which point the temperature was held constant for the duration of growth $(10 \mathrm{~min})$. The sulfur source was kept at $200{ }^{\circ} \mathrm{C}$ throughout. An overview of the process is illustrated in Fig. 1.

The process for $\mathrm{WS}_{2}$ growth was similar to that for $\mathrm{MoS}_{2}$, except that growth occurred at $900{ }^{\circ} \mathrm{C}$ instead of $700{ }^{\circ} \mathrm{C}$. One minor difference is a longer growth time for $\mathrm{WS}_{2}(15 \mathrm{~min}$ instead of $10 \mathrm{~min}$ ). The tungsten source was also prepared as is common for CVD growth of $\mathrm{WS}_{2}$, but this is different to the Mo source preparation. We simply placed $100 \mathrm{mg}$ of $\mathrm{WO}_{3}$ powder in a quartz boat and positioned it below the target substrate as described above. The placement of the sulfur source was identical in both cases, but we note that these growth procedures have not been fully optimized.

After TMDC growth, we characterized the results using optical microscopy and Raman spectroscopy. We used a Renishaw inVia Reflex micro-Raman spectrometer to collect Raman spectra and perform mapping. Excitation laser wavelengths were $514 \mathrm{~nm}$ for $\mathrm{MoS}_{2}$ and $488 \mathrm{~nm}$ for $\mathrm{WS}_{2}$ in order to avoid strong resonances at $514 \mathrm{~nm}^{17}$

\section{Results and discussion}

\section{1 $\mathrm{MoS}_{2}$ growth localized to patterned graphene}

The top row of Fig. 2(a)-(c) shows an optical micrograph of asgrown $\mathrm{MoS}_{2}$-graphene heterostructures and two superimposed Raman intensity maps. The Raman map in Fig. 2(b) shows the graphene 2D peak intensity, confirming graphene in the patterned rectangles. The other Raman map in Fig. 2(c) shows the integrated intensity of the two characteristic $\mathrm{MoS}_{2}$ peaks found between $370 \mathrm{~cm}^{-1}$ and $420 \mathrm{~cm}^{-1}$. Both Raman maps correspond to the same area, confirming $\mathrm{MoS}_{2}$ growth was directly atop, and confined by, patterned graphene.

In the upper panel of Fig. 2d, we show Raman spectra before and after $\mathrm{MoS}_{2}$ growth on graphene. These are labeled (i) and (ii), respectively. In addition to the graphene $\mathrm{G}$ and $2 \mathrm{D}$ peaks, ${ }^{18}$ additional peaks appear after $\mathrm{MoS}_{2}$ growth. Based on the decomposition and fitting shown in Fig. 2e, we attribute the small, emergent Raman peaks located at $1259 \mathrm{~cm}^{-1}$ and 1456 $\mathrm{cm}^{-1}$ to $\mathrm{C}-\mathrm{H}$ stretching and bending modes. ${ }^{19}$ Although these peaks only appear when sulfur is supplied, the positions and relative intensities more closely match peaks associated with $\mathrm{C}-\mathrm{H}$ than $\mathrm{C}-\mathrm{S}$ bonds. ${ }^{20}$ The origin of hydrogen, however, is not clear. We attribute the larger peaks at $1376 \mathrm{~cm}^{-1}, 1559 \mathrm{~cm}^{-1}$, and $2887 \mathrm{~cm}^{-1}$ to the $\mathrm{D}, \mathrm{G}$, and $2 \mathrm{D}$ peaks of amorphous carbon $\left(\mathrm{D}_{\mathrm{a}-\mathrm{C}}, \mathrm{G}_{\mathrm{a}-\mathrm{C}}, 2 \mathrm{D}_{\mathrm{a}-\mathrm{C}}\right){ }^{21}$

In the lower panel of Fig. 2d we show Raman spectra from graphene-on- $\mathrm{SiO}_{2} / \mathrm{Si}$ annealed at the $\mathrm{MoS}_{2}$ growth temperature $\left(700^{\circ} \mathrm{C}\right)$. When annealed under pure Ar (Fig. $\left.2 \mathrm{~d}(\mathrm{iii})\right)$, no peaks associated with a-C appear. When annealed in the presence of sulfur but in the absence of $\mathrm{MoO}_{3}$ (Fig. 2d(iv)), a-C peaks are clearly visible, and the spectrum is very similar to that of $\mathrm{MoS}_{2}$ grown on graphene (Fig. 2d(ii)). These results indicate that a-C formation is not simply due to elevated temperature, but the presence of sulfur at elevated temperature. ${ }^{20}$ In spite of this, the amount of a-C can be reduced by annealing in a sulfur environment at even higher temperature. This is shown in Fig. $2 \mathrm{~d}(\mathrm{v})$, which corresponds to $\mathrm{MoS}_{2}$ on graphene annealed at $900{ }^{\circ} \mathrm{C}$ for $20 \mathrm{~min}$ in the presence of sulfur. We note that the a-C Raman modes have considerably lower relative intensity, whereas the other peaks remain largely unchanged.

All spectra in Fig. 2d exhibit an upshift of the graphene G and $2 \mathrm{D}$ peaks, as well as broadening of the $2 \mathrm{D}$ peak relative to the as-transferred graphene. These changes suggest hole doping of the graphene occurs during thermal treatment ${ }^{22,23}$ and subsequent exposure to the atmosphere. ${ }^{24}$ 

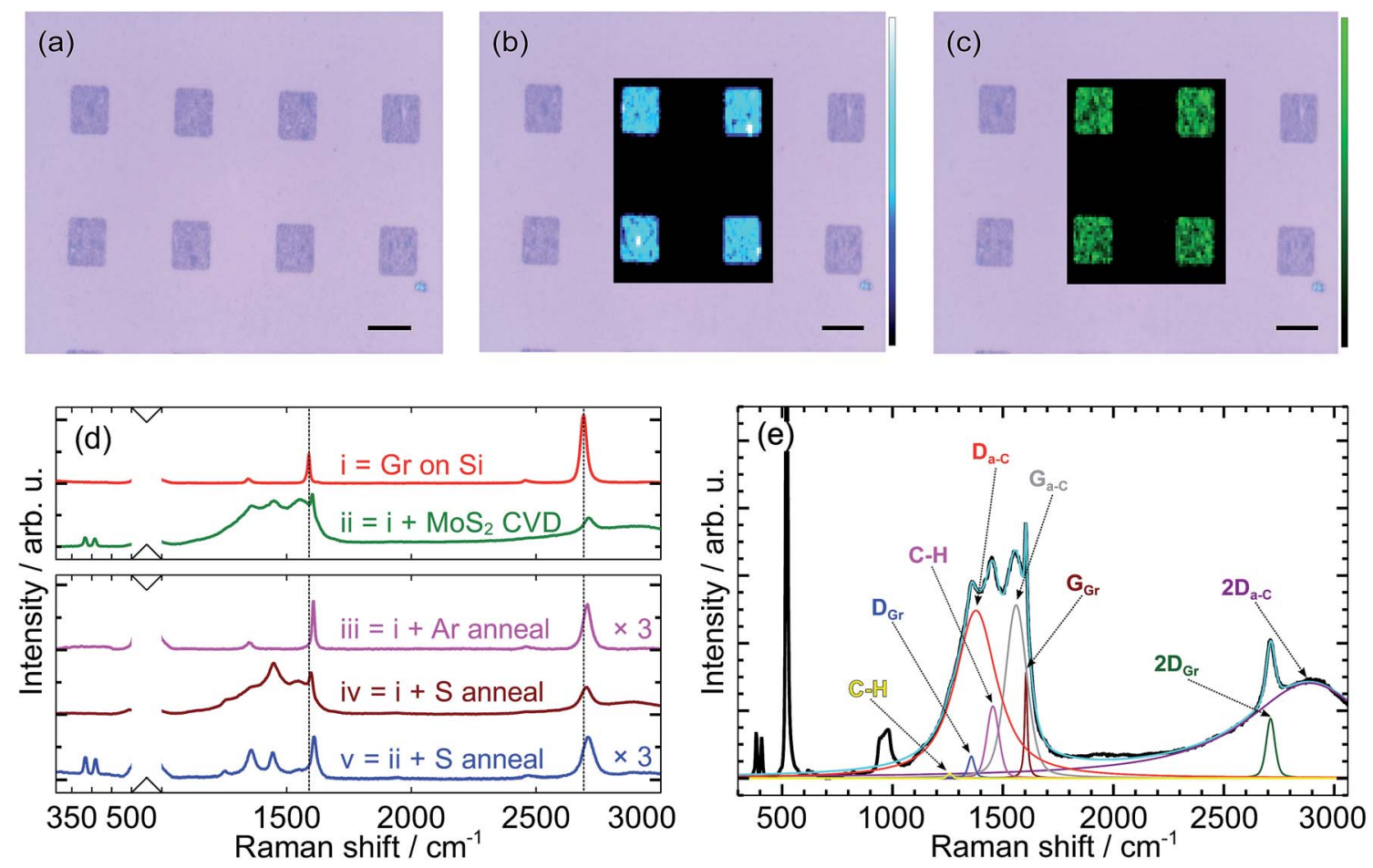

Fig. 2 (a) Optical micrograph of patterned graphene on $\mathrm{SiO}_{2} / \mathrm{Si}$. Scale bar corresponds to $20 \mu \mathrm{m}$. (b and c) Superimposed Raman maps showing $\mathrm{MoS}_{2}$ growth corresponds exactly to graphene pattern regions. (b) Intensity of graphene 2D peak at $2711 \mathrm{~cm}^{-1}$ and (c) integrated intensity of $\mathrm{MoS}_{2} E_{2 g}^{1}$ and $A_{1 g}$ peaks (from 370 to $420 \mathrm{~cm}^{-1}$ ). (d) Raman spectra of (i) graphene, (ii) $\mathrm{MoS}_{2}$ on graphene, and (iii-v) annealed versions of both. All spectra are normalized to the first-order silicon peak (hidden), and $\lambda_{\text {ex }}=514 \mathrm{~nm}$. (e) Decomposition of Raman spectrum after CVD of MoS 2 on graphene ((ii) in (d)).

Based on the positions of the $\mathrm{MoS}_{2} \mathrm{E}_{2 \mathrm{~g}}^{1}$ and $\mathrm{A}_{1 \mathrm{~g}}$ Raman modes, ${ }^{25,26}$ we find the $\mathrm{MoS}_{2}$ thickness ranges from two layers to five or more layers (bulk), with the majority being three or four layers of $\mathrm{MoS}_{2}$. We found the layer number to be very sensitive to the local $\mathrm{MoO}_{3}$ concentration. Near the center of the substrate, where the $\mathrm{MoO}_{3}$ concentration is highest (i.e., directly above the $\mathrm{MoO}_{3}$ source), we find $\mathrm{MoS}_{2}$ can nucleate and grow directly on the silicon wafer, but is predominantly bulk. Closer to the substrate edge, where the $\mathrm{MoO}_{3}$ concentration is lower, few-layer $\mathrm{MoS}_{2}$ selectively grows only on graphene. Our hypothesis for the selective growth on graphene is as follows. Since sulfur is introduced to the system from the beginning of the CVD process, it can satisfy dangling bonds present in graphene. When the growth temperature is reached and Mo is present, the attached sulfur atoms act as nucleation sites, leading to selective growth of $\mathrm{MoS}_{2}$ on graphene. This mechanism should also apply to other TMDCs under appropriate conditions, and the following results for $\mathrm{WS}_{2}$ suggest that to be the case.

\section{2 $\mathrm{WS}_{2}$ grown directly on graphene}

Fig. 3a shows an optical image of $\mathrm{WS}_{2}$ grown on graphene by CVD. We note that patterned graphene was not used here. Instead, we reduced the CVD time in order to avoid forming continuous graphene, and then grew $\mathrm{WS}_{2}$ atop that. Raman intensity maps of the graphene $2 \mathrm{D}$ and $\mathrm{WS}_{2} \mathrm{E}_{2 \mathrm{~g}}^{1}$ peaks are shown in Fig. $3 \mathrm{~b}$ and c. The mapped area corresponds to the dotted outline in Fig. 3a. Comparing the Raman maps with the optical micrograph, we see that the majority of the surface is covered by graphene, but only some of the graphene is covered by $\mathrm{WS}_{2}$. Darker regions in (a) are $\mathrm{WS}_{2}$ on top of graphene, whereas bright spots correspond to bulk $\mathrm{WS}_{2}$ (five or more layers). A few graphene voids are visible as slightly lighter patches. $\mathrm{WS}_{2}$ appears to grow right up to the edge of several of these voids, but does not extend out onto the $\mathrm{SiO}_{2}$ surface. This indicates that $\mathrm{WS}_{2}$ grows selectively on graphene, as was the case for $\mathrm{MoS}_{2}$.

In Fig. 3d we plot various Raman spectra for comparison. The top two spectra are from the Raman maps shown, whereas the bottom three spectra are from different processes but shown for comparison and clarification. Our analysis reveals several similarities with $\mathrm{MoS}_{2}$ grown on graphene. For example, we find $\mathrm{C}-\mathrm{H}$ peaks in the Raman spectra for both cases. Despite the presence of sulfur during $\mathrm{WS}_{2}$ growth at $900{ }^{\circ} \mathrm{C}$, no obvious a-C Raman peaks are found. This is consistent with our finding that annealing $\mathrm{MoS}_{2}$ at this temperature in a sulfur environment reduced the amount of a-C. Lowering the $\mathrm{WS}_{2}$ growth temperature from $900{ }^{\circ} \mathrm{C}$ to $850{ }^{\circ} \mathrm{C}$ increased coverage of $\mathrm{WS}_{2}$ on graphene, but the quality of graphene suffered (i.e., strong $\mathrm{C}-\mathrm{H}$ and a-C peaks appeared).

Based on the $\mathrm{E}_{2 \mathrm{~g}}^{1}$ and $\mathrm{A}_{1 \mathrm{~g}}$ peak separation, ${ }^{17}$ one significant difference between $\mathrm{WS}_{2}$ on graphene and $\mathrm{MoS}_{2}$ on graphene is that we find no few-layer regions of $\mathrm{WS}_{2}$. The $\mathrm{WS}_{2}$ grown on graphene is either monolayer (ML) or bulk (5+ layers). 

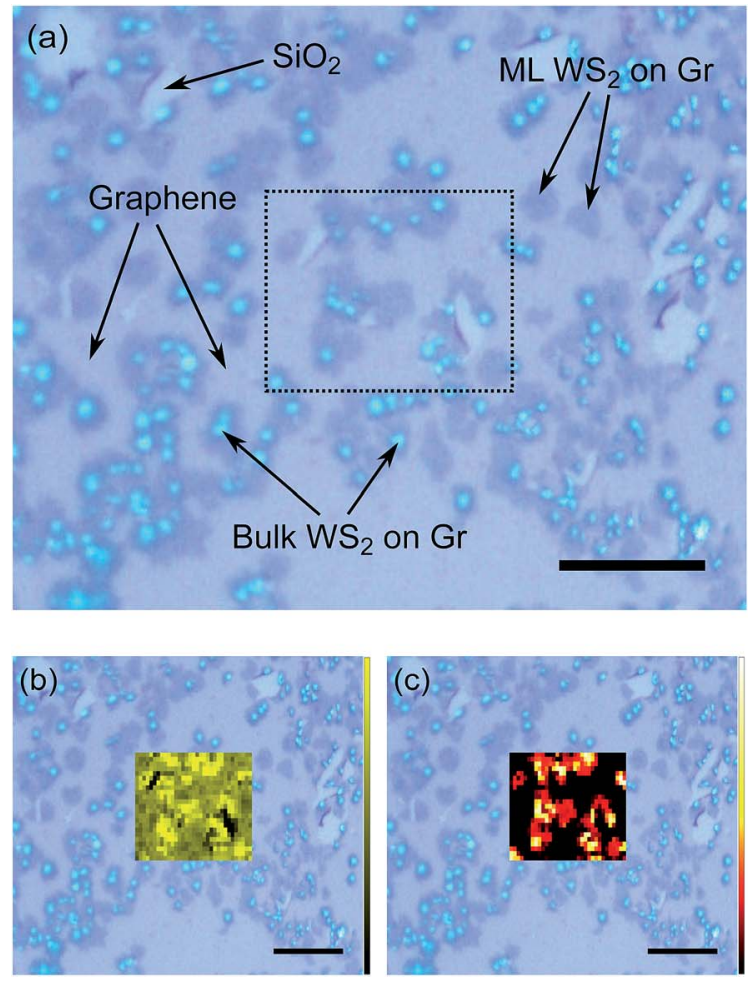

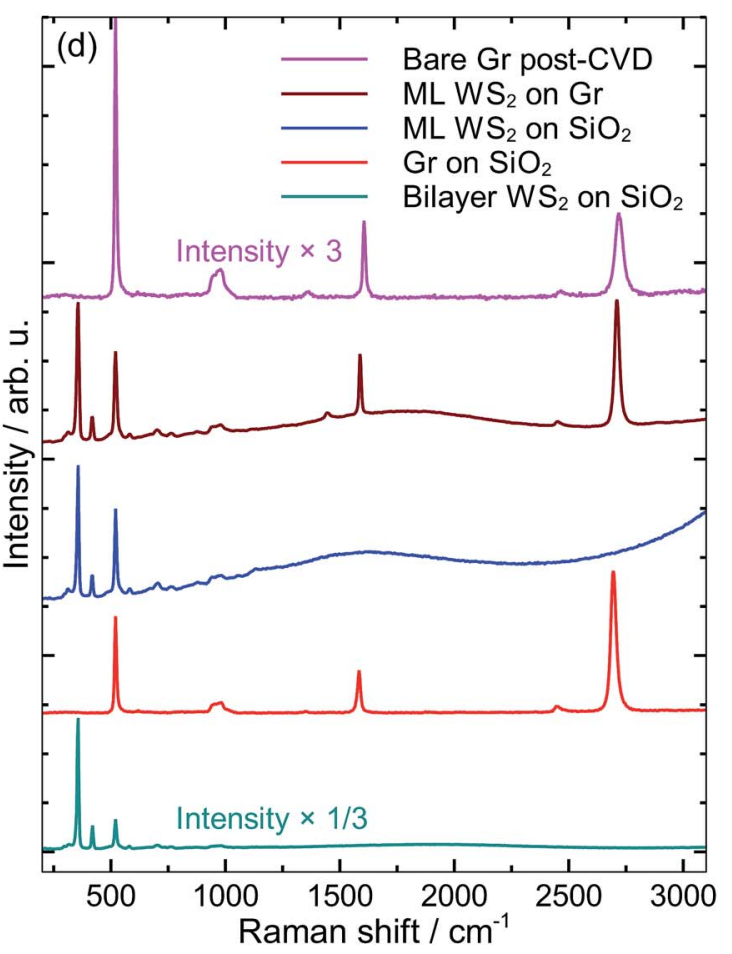

Fig. 3 (a) Optical micrograph of $W_{2} S_{2}$ grown on graphene. Dotted line denotes mapped area shown in (b and c). All scale bars are $20 \mu m$, and $\lambda_{\text {ex }}=$ $488 \mathrm{~nm}$. (b) Intensity map of graphene 2D peak at $2727 \mathrm{~cm}^{-1}$. (c) Intensity map of $E_{2 g}^{1}$ peak of monolayer $W_{2}$ at $354.9 \mathrm{~cm}^{-1}$. (d) Raman spectra of graphene and $\mathrm{WS}_{2}$ with different interfacial conditions. The topmost two spectra are from the map at left, whereas the lower three spectra are from different processes and shown for comparison. All spectra are normalized to the first-order silicon peak.

Importantly, we noticed small shifts in the $\mathrm{E}_{2 \mathrm{~g}}^{1}$ and $\mathrm{A}_{1 \mathrm{~g}}$ peak positions for $\mathrm{ML} \mathrm{WS}_{2}$ on graphene compared to $\mathrm{ML} \mathrm{WS}_{2}$ on $\mathrm{SiO}_{2} / \mathrm{Si}$. The $\mathrm{E}_{2 \mathrm{~g}}^{1}$ peak position shifts down slightly from 355.6 $\mathrm{cm}^{-1}$ to $355.1 \mathrm{~cm}^{-1}$, whereas the $\mathrm{A}_{1 \mathrm{~g}}$ peak shifts up slightly from $417.2 \mathrm{~cm}^{-1}$ to $417.6 \mathrm{~cm}^{-1}$. This is only slightly larger than the uncertainty of $0.3 \mathrm{~cm}^{-1}$, but the shift is consistent for more than 10 independent measurements. We find the $\mathrm{E}_{2 \mathrm{~g}}^{1}$ and $\mathrm{A}_{1 \mathrm{~g}}$ peak separation for monolayer $\mathrm{WS}_{2}$ on graphene to be $62.5 \mathrm{~cm}^{-1}$. This is slightly larger than the $61.6 \mathrm{~cm}^{-1}$ for $\mathrm{WS}_{2}$ on $\mathrm{SiO}_{2}$, yet still less than the $63.4 \mathrm{~cm}^{-1}$ that corresponds to bilayer $\mathrm{WS}_{2}$ on $\mathrm{SiO}_{2} \cdot{ }^{17}$

By comparing the $\mathrm{WS}_{2} \mathrm{E}_{2 \mathrm{~g}}^{1}$ and $\mathrm{A}_{1 \mathrm{~g}}$ peak intensities to the $\mathrm{Si}$ substrate peak at $520 \mathrm{~cm}^{-1}$, we can confirm the darker regions seen in Fig. 3a are indeed monolayer rather than bilayer $\mathrm{WS}_{2}$ on graphene. The presence of a fluorescence tail (onset visible near $3000 \mathrm{~cm}^{-1}$ ) is further evidence that the spectrum labeled "ML $\mathrm{WS}_{2}$ on $\mathrm{SiO}_{2}$ " is indeed monolayer. The absence of this tail for monolayer $\mathrm{WS}_{2}$ on graphene is due to ultrafast charge transfer to graphene, ${ }^{27}$ indicating a clean and sharp interface in the heterostructure. Moreover, the strong Raman signal suggests graphene quality remains high despite the high-temperature growth environment.

\section{Conclusion}

In conclusion, we report CVD growth of few-layer $\mathrm{MoS}_{2}$ directly atop patterned graphene in which the $\mathrm{MoS}_{2}$ is confined to the graphene region. The $\mathrm{MoS}_{2}$ covered the entire graphene pattern, demonstrating the ability to define the shape and location of vertically stacked 2D heterostructures prior to CVD. We also report $\mathrm{WS}_{2}$ selectively grown on transferred graphene. Unlike $\mathrm{MoS}_{2}$, coverage of $\mathrm{WS}_{2}$ was incomplete, and was found to be either bulk or monolayer. Obtaining complete coverage of $\mathrm{WS}_{2}$ should be a matter of finding appropriate growth conditions and is left for future study. For both $\mathrm{MoS}_{2}$ and $\mathrm{WS}_{2}$, we hypothesize that sulfur atoms that satisfy dangling bonds on the graphene act as nucleation sites, confining the growth location. Similar findings using two different TMDCs, despite considerably different growth conditions, suggests this graphene-templated selective growth may apply to TMDCs in general. We expect these results will facilitate batch fabrication of $2 \mathrm{D}$ heterostructure systems and devices.

\section{Acknowledgements}

This work was supported by Award FA9550-16-1-0188 from the Air Force Office of Scientific Research, and by the Innovative Micro-Programs Accelerating Collaboration in Themes (IMPACT) program funded by the Office of Vice President of Research and Economic Development at the University at Buffalo.

\section{References}

1 A. C. Ferrari, F. Bonaccorso, V. Fal'ko, K. S. Novoselov, S. Roche, P. Bøggild, S. Borini, F. H. L. Koppens, 
V. Palermo, N. Pugno, J. A. Garrido, R. Sordan, A. Bianco, L. Ballerini, M. Prato, E. Lidorikis, J. Kivioja, C. Marinelli, T. Ryhänen, A. Morpurgo, J. N. Coleman, V. Nicolosi, L. Colombo, A. Fert, M. Garcia-Hernandez, A. Bachtold, G. F. Schneider, F. Guinea, C. Dekker, M. Barbone, Z. Sun, C. Galiotis, A. N. Grigorenko, G. Konstantatos, A. Kis, M. Katsnelson, L. Vandersypen, A. Loiseau, V. Morandi, D. Neumaier, E. Treossi, V. Pellegrini, M. Polini, A. Tredicucci, G. M. Williams, B. H. Hong, J.-H. Ahn, J. M. Kim, H. Zirath, B. J. van Wees, H. van der Zant, L. Occhipinti, A. Di Matteo, I. A. Kinloch, T. Seyller, E. Quesnel, X. Feng, K. Teo, N. Rupesinghe, P. Hakonen, S. R. T. Neil, Q. Tannock, T. Löfwander and J. Kinaret, Nanoscale, 2015, 7, 4598-4810.

2 C. Chen, Z. Feng, Y. Feng, Y. Yue, C. Qin, D. Zhang and W. Feng, ACS Appl. Mater. Interfaces, 2016, 8, 19004-19011.

3 W. Zhang, C.-P. Chuu, J.-K. Huang, C.-H. Chen, M.-L. Tsai, Y.-H. Chang, C.-T. Liang, Y.-Z. Chen, Y.-L. Chueh, J.-H. He, M.-Y. Chou and L.-J. Li, Sci. Rep., 2014, 4, 3826.

4 K. Roy, M. Padmanabhan, S. Goswami, T. P. Sai, G. Ramalingam, S. Raghavan and A. Ghosh, Nat. Nanotechnol., 2013, 8, 826-830.

5 M. Zhao, Y. Ye, Y. Han, Y. Xia, H. Zhu, S. Wang, Y. Wang, D. A. Muller and X. Zhang, Nat. Nanotechnol., 2016, 11, 954-959.

6 T. Georgiou, R. Jalil, B. D. Belle, L. Britnell, R. V. Gorbachev, S. V. Morozov, Y.-J. Kim, A. Gholinia, S. J. Haigh, O. Makarovsky, L. Eaves, L. A. Ponomarenko, A. K. Geim, K. S. Novoselov and A. Mishchenko, Nat. Nanotechnol, 2013, 8, 100-103.

7 F. Withers, O. Del Pozo-Zamudio, A. Mishchenko, A. P. Rooney, A. Gholinia, K. Watanabe, T. Taniguchi, S. J. Haigh, A. K. Geim, A. I. Tartakovskii and K. S. Novoselov, Nat. Mater., 2015, 14, 301-306.

8 L. Britnell, R. M. Ribeiro, A. Eckmann, R. Jalil, B. D. Belle, A. Mishchenko, Y.-J. Kim, R. V. Gorbachev, T. Georgiou, S. V. Morozov, A. N. Grigorenko, A. K. Geim, C. Casiraghi, A. H. Castro Neto and K. S. Novoselov, Science, 2013, 340, 1311-1314.

9 Y. Shi, W. Zhou, A.-Y. Lu, W. Fang, Y.-H. Lee, A. L. Hsu, S. M. Kim, K. K. Kim, H. Y. Yang, L.-J. Li, J.-C. Idrobo and J. Kong, Nano Lett., 2012, 12, 2784-2791.

10 C. E. Giusca, I. Rungger, V. Panchal, C. Melios, Z. Lin, Y.-C. Lin, E. Kahn, A. L. Elías, J. A. Robinson, M. Terrones and O. Kazakova, ACS Nano, 2016, 10, 7840-7846.
11 A. Rossi, H. Büch, C. D. Rienzo, V. Miseikis, D. Convertino, A. Al-Temimy, V. Voliani, M. Gemmi, V. Piazza and C. Coletti, 2D Materials, 2016, 3, 031013.

12 Y.-C. Lin, C.-Y. S. Chang, R. K. Ghosh, J. Li, H. Zhu, R. Addou, B. Diaconescu, T. Ohta, X. Peng, N. Lu, M. J. Kim, J. T. Robinson, R. M. Wallace, T. S. Mayer, S. Datta, L.-J. Li and J. A. Robinson, Nano Lett., 2014, 14, 6936-6941.

13 D. Pierucci, H. Henck, C. H. Naylor, H. Sediri, E. Lhuillier, A. Balan, J. E. Rault, Y. J. Dappe, F. Bertran, P. L. Fèvre, A. T. C. Johnson and A. Ouerghi, Sci. Rep., 2016, 6, 26656.

14 X. Li, Y. Zhu, W. Cai, M. Borysiak, B. Han, D. Chen, R. D. Piner, L. Colombo and R. S. Ruoff, Nano Lett., 2009, 9, 4359-4363.

15 G. B. Barin, Y. Song, I. F. Gimenez, A. G. Souza Filho, L. S. Barreto and J. Kong, Carbon, 2015, 84, 82-90.

16 Z. Lin, Y. Zhao, C. Zhou, R. Zhong, X. Wang, Y. H. Tsang and Y. Chai, Sci. Rep., 2015, 5, 18596.

17 A. Berkdemir, H. R. Gutiérrez, A. R. Botello-Méndez, N. Perea-López, A. L. Elías, C.-I. Chia, B. Wang, V. H. Crespi, F. López-Urías, J.-C. Charlier, H. Terrones and M. Terrones, Sci. Rep., 2013, 3, 1-8.

18 A. C. Ferrari, J. C. Meyer, V. Scardaci, C. Casiraghi, M. Lazzeri, F. Mauri, S. Piscanec, D. Jiang, K. S. Novoselov, S. Roth and A. K. Geim, Phys. Rev. Lett., 2006, 97, 187401.

19 H. Wu, J. V. Volponi, A. E. Oliver, A. N. Parikh, B. A. Simmons and S. Singh, Proc. Natl. Acad. Sci. U. S. A., 2011, 108, 38093814 .

20 L. Zhang, L. Ji, P.-A. Glans, Y. Zhang, J. Zhu and J. Guo, Phys. Chem. Chem. Phys., 2012, 14, 13670-13675.

21 J. Hong, M. K. Park, E. J. Lee, D. Lee, D. S. Hwang and S. Ryu, Sci. Rep., 2013, 3, 2700.

22 S. Ryu, L. Liu, S. Berciaud, Y.-J. Yu, H. Liu, P. Kim, G. W. Flynn and L. E. Brus, Nano Lett., 2010, 10, 4944-4951.

23 J. E. Lee, G. Ahn, J. Shim, Y. S. Lee and S. Ryu, Nat. Commun., 2012, 3, 1024.

24 L. Liu, S. Ryu, M. R. Tomasik, E. Stolyarova, N. Jung, M. S. Hybertsen, M. L. Steigerwald, L. E. Brus and G. W. Flynn, Nano Lett., 2008, 8, 1965-1970.

25 C. Lee, H. Yan, L. E. Brus, T. F. Heinz, J. Hone and S. Ryu, ACS Nano, 2010, 4, 2695-2700.

26 H. Li, Q. Zhang, C. C. R. Yap, B. K. Tay, T. H. T. Edwin, A. Olivier and D. Baillargeat, Adv. Funct. Mater., 2012, 22, 1385-1390.

27 J. He, N. Kumar, M. Z. Bellus, H.-Y. Chiu, D. He, Y. Wang and H. Zhao, Nat. Commun., 2014, 5, 5622. 